\title{
MUSCLE BULK, STRENGTH AND PROPRIOCEPTIVE DIFFERENCE BETWEEN ANTERIOR CRUCIATE LIGAMENT DEFICIENT AND NORMAL KNEE
}

\author{
Kalimuthu $\mathrm{M}^{1,2^{*}}$, Mokhtar $\mathrm{AH}^{1,2}$ \\ 1 Sports Medicine Clinic, University of Malaya Medical Center, Kuala Lumpur, Malaysia \\ 2 Sports Medicine Department, University of Malaya Medical Center, Kuala Lumpur, Malaysia
}

\section{Correspondence:}

Dr MohanaKumar Kalimuthu

Sports Medicine Department,

5th floor, South Tower,

University of Malaya Medical Center,

59100, Kuala Lumpur, Malaysia

Tel: (603) $79674968 / 79677522$

Fax: (603) 79677511

E mail address: mohanakumar16@yahoo.com

\begin{abstract}
The anterior cruciate ligament ( $\mathrm{ACL}$ ) deficient knee has been subjected to various research and many conclusions have been drawn to the loss of function in relation to it. ACL reconstruction is also very commonly done as this ligament damage is the most common injury in sports. Muscle strength and proprioception have been postulated to be affected alongside this injury. We conducted a cross sectional study in 19 patients who have ruptured their ACL. We measured their muscle strength, thigh muscle bulk and proprioception in comparison to their contralateral normal knee. The Biodex Isokinetic machine was used to measure the muscle strength and proprioception. The thigh circumference was measured manually using a measuring tape. There was significant reduction of muscle strength and thigh circumference in the ACL deficient limb as compared to the normal side, $p<0.05$. The affected knee also showed reduction in proprioceptive ability at 70 degree angle $(z$ score $-2.17, p<0.05)$, but there was no significant reduction at the other angles. These findings are important as the data show that there is reduction in muscle strength and proprioceptive ability in an ACL deficient knee; hence, rehabilitation addressing these two areas should be emphasized pre-surgery.
\end{abstract}

Keywords: Anterior cruciate ligament, circumference, position, power, sense, thigh

\section{Introduction}

The anterior cruciate ligament injury is a common injury that occurs in individuals active primarily in sports and recreational activities (1). The injury causes loss of muscle function (as the strength profile of the quadriceps and hamstrings are affected by the neural effect of the ACL injury), and disuse of the limb. However, the main focus has always been investigation of thigh muscle weakness post reconstruction (2). Comparison of muscle group (thigh flexors and extensors) strength difference, and donor site comparison with reference to strength deficit, seem to be a heatedly debated topic. A study showed that the quadriceps strength deficit was less than that of the hamstring, despite the use of a hamstring graft (3).

Besides muscle strength, joint proprioception has also been the subject of interest over the past 100 years.
Proprioception, in the early years termed "kineasthesis", means appreciation of joint position sense via afferent signals from skin, muscle and joints (4). The ACL deficient knee has been studied and demonstrated to have diminished proprioception, delayed reflex responses of the muscle and altered neuromuscular control (5). Proprioception gives information regarding the movement and the position of our leg, which is important for muscular control (6). It was stated that the mechanoreceptors responsible for proprioception constitute $1-2 \%$ of the bulk of the ACL tissue and are situated near the tibial and the femoral insertions. We hypothesize that patients with $\mathrm{ACL}$ deficient knee will have less muscle strength than the contralateral normal knee. Recently, a lot of importance has been placed on the role of surgery in the treatment of $\mathrm{ACL}$ rupture. Many questions have been raised with regards to the loss of muscle strength and the role of 
proprioception after the injury. Many surgical techniques and how they affect the restoration of normal function of the knee have triggered investigations exploring methods to improve the eventual results of surgery, and that of pre-surgical intervention. Several studies performed on ACL injury, however, were conducted outside Malaysia, and most studies concentrated on muscle strength and proprioception changes postoperatively, rather than preoperatively (1,7-9).

In this study, the differences in quadriceps and hamstring muscle bulk and strength between the $\mathrm{ACL}$ deficient and unaffected knees of the same individuals were investigated. Additionally, proprioceptive changes between the injured and uninjured knees were also explored.

\section{Methods}

A cross sectional study on the ACL deficient knee, as well as on the contralateral knee, of each patient, was done to compare the bulk of thigh musculature (quadriceps, hamstrings), muscle strength and proprioception. The study was conducted at the Sports Medicine Clinic, University of Malaya Medical Center between January 2012 and August 2014.

Male patients of 17-40 years old, with ACL injury, were invited to participate in this research. The participants in this study were clinically diagnosed with complete tear of the $A C L$, and the diagnosis confirmed with magnetic resonance imaging. Patients who had contralateral knee injuries, previous knee injuries or surgeries, persisting pain and inflammation of the knees, were excluded from this study.

Information about the study, and the procedures involved, were explained to potential participants, and the study information sheet distributed to them. Upon enrollment, information on socio-demographic information, including date of birth, gender, ethnicity, date of injury and date of diagnosis were gathered. Following this, the physical assessment of the quadriceps and hamstring muscle bulk, muscle strength and joint proprioception were performed. The quadriceps and hamstring bulk of the $\mathrm{ACL}$ deficient knee were measured first in all participants. This was performed using a measuring tape (to the nearest decimal point) with patients in supine position, exposed appropriately with the knee flexed to about 10 degrees. The thigh circumference was measured at 5, 10 and 15 $\mathrm{cm}$ proximal to the superior pole of the patella. The same investigator then repeated the measurement on the contralateral normal knee.

The quadriceps and hamstring strength was measured using the Biodex Isokinetic Dynamometer (Biodex System 4 Pro, New York). The Biodex has been shown to be a reliable instrument to measure the isokinetic peak torque in humans (10). Each patient was asked to warm up on a stationary bicycle for about 10 minutes before the test. The patient was strapped in and secured at the shoulders, chest and hips. Device set-up and subject positioning were as per the manufacturer's guidelines (Biodex system 3 ) which were similar to methods conducted in previous researches (11). Isokinetic testing was performed at 120 degrees/sec followed by 180 degrees/sec. These angles were chosen as they do not add extra stress to the ACL deficient knees (3). Three trials were allowed before the actual test. The angles were tested with a 30 second rest in between. The order for testing the injured knee and the contralateral normal knee was carried out randomly.

Finally, proprioception was tested at 3 separate angles. The joint position sense was tested at 45,60 and 70 degrees $(5,11-13)$ of knee flexion. The patient was seated on the Biodex, similar to the one used before, and then the knee, at random order, was moved to 45 degrees from 90 degree flexion and kept in the same position for 5 seconds. The patient was asked to remember this angle, and then the knee returned to flexion at 90 degrees. The patient, then with his eyes closed (to avoid visual input) attempted to reproduce the 45 degree angle. The mean degree of error was documented and used for analysis. The patient was given three attempts. After that, the other two angles (60 and 70 degrees) were tested in similar fashion (14). Our hypothesis was that proprioception will be altered in $\mathrm{ACL}$ deficient knee as compared to the contralateral normal side. This theory has been proven in previous tests $(4,15)$.

The data analysis was carried out using IBM SPSS Statistical Package 21, Armonk NY, and $p<0.05$ was considered statistically significant. The variables collected from the patients were calculated as means \pm standard deviation. Most of the variables were tested with Shapiro Wilk test and they demonstrated normal distribution $(p>0.05)$. The data that did not follow normal distribution were calculated using the median $\pm I Q R$, and tested with Wilcoxon Signed Ranks test.

\section{Results}

A total of 19 male patients between the age of 17 and 40 years (mean age 27.53) participated in this research. 16 were Malay and the other 3 were Indians. Eleven (57.9\%) patients had complete rupture of $A C L$ diagnosed on the right side, and eight (42.1\%) with ruptured $A C L$ diagnosed on the left. The mean height and weight of the subject population was $169.0 \mathrm{~cm}$ and $73.34 \mathrm{~kg}$ respectively. The mean BMI was 25.78. The results of the study are presented below. Tables 1, 2 and 3 show the peak torque, thigh circumference and proprioception between the injured versus the uninjured knee respectively. 
Table 1: Peak torque of the injured and uninjured knee at two different speeds

\begin{tabular}{|l|l|l|l|l|l|}
\hline Peak torque $(\mathrm{Nm})$ & $\begin{array}{l}\text { Injured } \\
\text { Mean } \pm \text { SD }\end{array}$ & $\begin{array}{l}\text { Uninjured } \\
\text { Mean } \pm \text { SD }\end{array}$ & T score/z score* & df & P value \\
\hline 120 flexion & $69.8 \pm 43.1$ & $98.9 \pm 53.6$ & $-3.06 *$ & - & $<0.001$ \\
120 extension & $102.66 \pm 39.5$ & $138.50 \pm 35.64$ & -7.3 & 18 & $<0.001$ \\
180 flexion & $50.98 \pm 19.62$ & $66.58 \pm 19.55$ & -4.6 & 18 & $<0.001$ \\
180 extension & $79.68 \pm 30.54$ & $103.31 \pm 32.0$ & -4.9 & 18 & $<0.001$ \\
\hline
\end{tabular}

df: degree of freedom

SD: standard deviation

$p<0.05$ is considered statistically significant

Table 2: Thigh circumference of the injured and uninjured knee

\begin{tabular}{|l|l|l|l|l|l|}
\hline Thigh circumference $(\mathrm{cm})$ & $\begin{array}{l}\text { Injured } \\
\text { Mean } \pm \text { SD }\end{array}$ & $\begin{array}{l}\text { Uninjured } \\
\text { Mean } \pm \text { SD }\end{array}$ & T score & df & P value \\
\hline 5 & $42.58 \pm 4.17$ & $44.26 \pm 4.29$ & -6.9 & 18 & $<0.001$ \\
10 & $47.74 \pm 4.63$ & $49.26 \pm 4.62$ & -8.6 & 18 & $<0.001$ \\
15 & $51.74 \pm 4.83$ & $53.74 \pm 5.02$ & 10.7 & 18 & $<0.001$ \\
\hline
\end{tabular}

df: degree of freedom

SD: standard deviation

$p<0.05$ is considered statistically significant

Table 3: Proprioceptive findings in the injured and uninjured knee

\begin{tabular}{|l|l|l|l|l|l|}
\hline Proprioception & $\begin{array}{l}\text { Injured } \\
\text { Mean } \pm \text { SD }\end{array}$ & $\begin{array}{l}\text { Non injured } \\
\text { Mean } \pm \text { SD }\end{array}$ & T score/ z score* & df & P value \\
\hline 45 & $5.25 \pm 2.92$ & $4.53 \pm 2.72$ & 0.9 & 18 & 0.39 \\
60 & $2.7 \pm 3.2$ & $4.4 \pm 4$ & $-1.22^{*}$ & - & 0.22 \\
70 & $1.7 \pm 1.6$ & $3.2 \pm 3.8$ & $-2.17^{*}$ & - & 0.03 \\
\hline
\end{tabular}

df: degree of freedom

SD: standard deviation

$p<0.05$ is considered statistically significant

As shown above, there is significant difference in peak torque between the injured and the uninjured knee at both 120 and 180 degrees/sec, less peak torque seen in the $\mathrm{ACL}$ deficient side. The thigh circumferences at 5, 10 and $15 \mathrm{~cm}$ of the injured side were significantly less; $p<0.05$ compared to the uninjured side. There were no significant differences in knee proprioception assessed at angles of 45 and 60 degrees; between the injured and uninjured side. However, proprioception assessment at 70 degrees of knee flexion showed a significant difference between the injured and uninjured side.

\section{Discussion}

The aim of this study was to investigate the difference in the quadriceps and hamstring strength, muscle bulk and knee joint proprioception in a normal compared to an ACL deficient knee. Our result showed that there was a significant difference with regards to hamstring and quadriceps strength and bulk; however, with regards to proprioception, there was a difference but not in all the angles tested. The subjects were tested on the Biodex at 120 and 180 degrees (flexion and extension) and all the tests showed that there was a significant difference between the muscle strength of the injured and the uninjured knee with $p$ values less than 0.05 . These findings are in agreement with those in reported literature (2). Previous studies, however, focused on post-surgery thigh strength measurements $(1,16)$. Only a few had obtained pre-surgery data, and most had comparisons to chronic ACL tears (18 months). Those studies examining patients with a duration of injury are uncommon. $A C L$ injury has been proven to cause atrophy of the quadriceps and hamstrings. A previous study reported $8 \%$ reduction in size in cross sectional area of the thigh in ACL deficient limb (17). Our study revealed a significant difference in thigh cross section, measured manually, and consistent with findings of previous studies. The proprioception measurements revealed a significant difference in the 70 degree evaluation $(p<0.05)$ but the 45 and 60 degree evaluations were not significant $(p>0.05)$. 
We used a Biodex machine in this research. This method is purely computer controlled and therefore reduces the error observed in human dependent methods. However, a person who is properly trained is needed to operate the machine. The reason for the significant difference has been postulated to be due to a direct inhibition of the quadriceps by knee effusion and capsular distension (6).

The conflicting results of the proprioception tests may be attributed to several reasons. Firstly, the proprioception tests were carried out immediately after testing the peak torque in the knee. Muscular fatigue has been postulated to affect proprioception (18-21). The subjects may not have been able to reproduce the desired position due to muscle fatigue but as the effect wore off, at 70 degree angle - tests were performed at 45, 60 and 70 degrees in that order - the performance accordingly produced the desired result. Secondly, the subjects may have taken time to understand the instructions of the tests and to familiarize themselves with the technique of assessment. Therefore, they needed several attempts before complying with the instructions. Finally, as mentioned earlier, the other structures (i.e. collateral ligaments, meniscus) that were injured along with the ACL may have caused some alterations in proprioception, thus affecting the outcome of the tests.

Several other limitations need to be addressed in the current study. Firstly, included in this study is the heterogenicity of $A C L$ injuries-for instance, the majority of patients in this study had a combination of complete $\mathrm{ACL}$ rupture with other structural damages (like meniscus and collateral ligament injuries). A more conclusive data could have been obtained from patients with isolated $A C L$ injury (22). The other limitation of this method is that only manual measurement was used; if the usage of MRI was included, we could obtain the actual size of the musculature and also identify the group of muscles involved and these data could be discussed. Previous reports suggest that the quadriceps atrophies more significantly compared to the hamstrings $(23,24)$. The patients were also examined at various time intervals after the injury, ranging from 4 weeks to 24 months. Therefore, it was difficult to quantify the rate of muscle atrophy with time, both in the acute or chronic stage. Each of the measurement points (eg. 5,10,15 $\mathrm{cm}$ ) represents groups of muscles as reported in a previous study (25). Finally, it is advisable to test the muscle strength and proprioception at different sessions, as muscle fatigue may interfere with accurate data collection.

\section{Conclusion}

This research shows that rupture of the anterior cruciate ligament causes reduction of muscular strength and bulk, and it also affects proprioception. Previous studies have reported similar results after surgery has been performed, and some patients take years before they develop muscle strength comparable to the contralateral normal knee. More emphasis has to be put on pre surgical rehabilitation. The patient should be put on muscle strength training and also proprioception with balance training (e.g. perturbation training, elastic band exercises, body weight training). This can help reduce the loss of muscle strength and bulk after injury. However, caution must be given to avoid certain exercises as it can cause further damage (e.g. chondral injury) to the $A C L$ deficient knee. Examples of exercises to be avoided are the open chain type of muscle strength training for the lower limb.

More research has to be done after injury to the $\mathrm{ACL}$, before the surgical intervention period, as rehabilitation after knee injury may help patients to have a better surgical outcome. Patients are normally concerned about how the surgery is going to help them lead an active lifestyle. With proper rehabilitation pre- and post-surgery, this can be obtained.

\section{Acknowledgement}

I would like to thank the participants who agreed to take part in this research. Without their consent and cooperation, this work would not have been made possible. Their approval to allow me to put them on the Isokinetic machine which requires a fair amount of effort on their side has given me an opportunity to collect much valued data. I also would love to extend my highest gratitude to my supervisor, Associate Professor Dr Abdul Halim bin Mokhtar for allowing me to pursue this topic for my research.

Without his guidance it would have been extremely difficult for me to plan and execute this research. It is also imperative for me to mention that this is my first attempt in a research and his guidance has been of great value. I also wish to thank the head of the Sports Medicine department University Malaya, Dr Mohd Nahar Azmi bin Mohammed for giving me an opportunity to carry out this research.

It is my pleasure to express my gratitude to $\mathrm{Dr}$ Mohd Shariff bin A Hamid for lending his valuable knowledge in statistics. Finally, I would like to thank my colleagues, Dr Moriffin bin Mahpis, Dr Jerri Ling Chui Yun, Dr Au Yong Pui San, Dr Rahmani Jaafar and Dr Kavitha Mahadevan for their moral support.

\section{References}

1. Hiemstra LA, Webber S, MacDONALD PB, Kriellaars DJ. Knee strength deficits after hamstring tendon and patellar tendon anterior cruciate ligament reconstruction. Med. Sci. Sports Exerc. 2000; 32(8):1472-1479.

2. Tsepis E, Vagenas G, Ristanis S, Georgoulis AD. Thigh Muscle Weakness in ACL-deficient knees persists without structured rehabilitation: Clin. Orthop. 2006; 450:211-218. doi:10.1097/01. blo.0000223977.98712.30.

3. Keays S, Bullock-Saxton J, Keays A, Newcombe P. Muscle strength and function before and after anterior cruciate ligament reconstruction using semitendonosus and gracilis. The Knee 2001; 8(3):229-234. 
4. Corrigan JP, Cashman WF, Brady MP. Proprioception in the cruciate deficient knee. J. Bone Joint Surg. Br. 1992; 74(2):247-250.

5. Subasi SS, Gelecek N, Aksakoglu G. Effects of different warm-up periods on knee proprioception and balance in healthy young individuals. J. Sport Rehabil. 2008; 17(2):186.

6. Fremerey RW, Lobenhoffer P, Zeichen J, Skutek $M$, Bosch $U$, Tscherne H. Proprioception after rehabilitation and reconstruction in knees with deficiency of the anterior cruciate ligament A Prospective, Longitudinal Study. J. Bone Joint Surg. Br. 2000; 82(6):801-806.

7. Goradia VK, Grana WA, Pearson SE. Factors Associated With Decreased Muscle Strength After Anterior Cruciate Ligament Reconstruction With Hamstring Tendon Grafts. Arthrosc. J. Arthrosc. Relat. Surg. 2006; 22(1):80.e1-80.e15. doi:10.1016/j. arthro.2005.10.012.

8. Kobayashi A, Higuchi H, Terauchi M, Kobayashi F, Kimura M, Takagishi K. Muscle performance after anterior cruciate ligament reconstruction. Int. Orthop. 2004; 28(1):48-51. doi:10.1007/s00264-0030502-5.

9. Barrett D. Proprioception and function after anterior cruciate reconstruction. J. Bone Joint Surg. Br. 1991; 73(5):833-837.

10. Giagazoglou P, Amiridis IG, Zafeiridis A, Thimara M, Kouvelioti V, Kellis E. Static balance control and lower limb strength in blind and sighted women. Eur. J. Appl. Physiol. 2009; 107(5):571-579.

11. Daneshjoo A, Mokhtar AH, Rahnama N, Yusof A. The effects of comprehensive warm-up programs on proprioception, static and dynamic balance on male soccer players. PloS One 2012; 7(12):e51568.

12. Tsiganos G, Kalamvoki E, Smirniotis J. Effect of the chronically unstable ankle on knee joint position sense. Isokinet. Exerc. Sci. 2008; 16(2):75-79.

13. Akseki D, Akkaya G, Erduran M, Pinar H. Proprioception of the knee joint in patellofemoral pain syndrome. Acta Orthop. Traumatol. Turc. 2004; 42(5):316-321.

14. Katayama $M$, Higuchi $H$, Kimura $M$, et al. Proprioception and performance after anterior cruciate ligament rupture. Int. Orthop. 2004; 28(5):278-281. doi:10.1007/s00264-004-0583-9.

15. Pap G, Machner A, Nebelung W, Awiszus F. Detailed analysis of proprioception in normal and ACL-deficient knees. J. Bone Joint Surg. Br. 1999; 81(5):764-768.

16. Anderson JL, Lamb SE, Barker KL, Davies S, Dodd CA, Beard DJ. Changes in muscle torque following anterior cruciate ligament reconstruction: a comparison between hamstrings and patella tendon graft procedures on 45 patients. Acta Orthop. 2002; 73(5):546-552.

17. Arangio GA, Chen C, Kalady M, Reed III JF. Thigh muscle size and strength after anterior cruciate ligament reconstruction and rehabilitation. J. Orthop. Sports Phys. Ther. 1997; 26(5):238-243.
18. Changela PK, Selvamani K. A Study to Evaluate the effect of Fatigue on Knee Joint Proprioception and Balance in Healthy Individuals. 2013; 2(3):1-4.

19. Skinner H, Wyatt M, Hodgdon J, Conard D, Barrack R. Effect of fatigue on joint position sense of the knee. J. Orthop. Res. 1986; 4(1):112-118.

20. Lattanizio P-J, Petrella RJ, Sproule JR, Fowler PJ. Effects of fatigue on knee proprioception. Clin. J. Sport Med. 1997; 7(1):22-27.

21. Ali Y, Farzaneh $\mathrm{G}$, Homayoon A. The effect of fatigue on the ankle and knee proprioception and dynamic control of posture. Int. J. Sports Sci. Fit. 2013; 3(2):139-48.

22. Roberts D, Ageberg E, Andersson G, Fridén T. Clinical measurements of proprioception, muscle strength and laxity in relation to function in the ACL-injured knee. Knee Surg. Sports Traumatol. Arthrosc. 2007; 15(1):9-16. doi:10.1007/s00167-006-0128-4.

23. Lorentzon R, Elmqvist L-G, Sjostrom $M$, Fagerlund $M$, Fugl-Meyer AR. Thigh musculature in relation to chronic anterior cruciate ligament tear: muscle size, morphology, and mechanical output before reconstruction. Am. J. Sports Med. 1989;17(3):423429.

24. Kilic BA, Dingil O, Erkula G, Elmas C, Erdogan D, Atik OS. Evaluation of the muscles around the knee in rabbits whose anterior cruciate and/or medial collateral ligaments were dissected. Arch. Orthop. Trauma Surg. 2004; 124(9):626-630.

25. Nicholas J, Taylor F, Buckingham R, Ottonello D. Measurement of circumference of the knee with ordinary tape measure. Ann. Rheum. Dis. 1976; 35(3):282-284. 